Pacific Journal of Mathematic 


\title{
HOMOTOPY CLASSIFICATION OF LENS SPACES FOR ONE-RELATOR GROUPS WITH TORSION
}

\author{
SUSHIL JAJODIA
}

Let $\Xi$ be a one-relator group with presentation $\mathscr{R}=$ $\left(x_{1}, \cdots, x_{n}: R^{p}\right)$ where $R$ is not a proper power and $p \geqq 2$. Then given any integer $q$, relatively prime to $p$, we can construct the Lens space $\mathscr{L}(p, q)$ for $\Xi$ from the cellular model $C(\mathscr{R})$ of the presentation $\mathscr{R}$ by attaching a 3-cell via the attaching map $R^{q}-1$, which generates the ideal $Z \Xi(R-1) \approx \pi_{2}(C(\mathscr{R}))$. In this paper we classify these Lens spaces up to homotopy type. We also discuss the noncancellation aspect of these Lens spaces.

Introduction. In this paper we are interested in Lens spaces for one-relator groups with torsion. Given relatively prime integers $p$ and $q$, with $p \geqq 2$, we have the ordinary Lens space $L(p, q)$ with fundamental group finite cyclic of order $p$. The 2-skeleton of $L(p, q)$ is the cellular model $C(\mathscr{R})$ of the presentation

$$
\mathscr{R}=\left(x: x^{p}\right)
$$

and $L(q, p)$ is obtained from its 2-skeleton by attaching a 3-cell via the attaching map $x^{q}-1$, which generates the ideal $Z Z_{p}(x-1) \approx$ $\pi_{2}(C(\mathscr{R}))$. The cellular chain complex of the universal covering $\widetilde{L}(p, q)$ of $L(p, q)$ is given by

$$
C_{*}(L(p, q)): Z Z_{p} \stackrel{x^{q}-1}{\longrightarrow} Z Z_{p} \stackrel{1+x+\cdots+x^{p-1}}{\longrightarrow} Z Z_{p} \stackrel{x-1}{\longrightarrow} Z Z_{p}
$$

where $x$ is a generator of the cyclic group $Z_{p}$. J. H. C. Whitehead [11] has shown that $L(p, q)$ and $L(p, r)$ have the same homotopy type if and only if $q r$ or $-q r$ is a quadratic residue $\bmod p$. We consider the following analogue: Let $\Xi$ be a one-relator group with presentation

$$
\mathscr{R}=\left(x_{1}, \cdots, x_{n}: R^{p}\right)
$$

where $R$ is not a proper power and $p \geqq 2$. Then given any integer $q$, relatively prime to $p$, we can construct the Lens space $\mathscr{L}(p, q)$ obtained from the cellular model $C(\mathscr{R})$ of the presentation $R$ by attaching a 3-cell via the attaching map $R^{q}-1$, which generates the ideal $Z E(R-1) \approx \pi_{2}(C(\mathscr{R}))$. Clearly the fundamental group of $\mathscr{L}(p, q)$ is isomorphic to $\Xi$. The cellular chain complex for the universal covering $\tilde{\mathscr{L}}(p, q)$ of the Lens space $\mathscr{L}(p, q)$ is given by

$$
C_{*}(\tilde{\mathscr{L}}(p, q)): Z \Xi \stackrel{R^{q}-1}{\longrightarrow} Z \Xi \stackrel{\partial_{2}}{\longrightarrow}(Z \Xi)^{n} \stackrel{\partial_{1}}{\longrightarrow} Z \Xi
$$


where $\partial_{1}=\left(x_{1}-1, \cdots, x_{n}-1\right)$ and $\partial_{2}$ is Jacobian matrix of the presentation $\mathscr{R}$ described in the free differential calculus of R. H. Fox [2].

DEFinition. An element $R$ of free group $F$ generated by $x_{1}, \cdots, x_{n}$ is primitive if it is a member of a free basis for $F$.

THEOREM 1. Let $\Xi$ have presentation (1) with single relator $R^{p}$ a power of a primitive element $R$ of $F$. Then two Lens spaces $\mathscr{L}(p, q)$ and $\mathscr{L}(p, r)$ for $\Xi$ have the same homotopy type if and only if $q r$ or $-q r$ is a quadratic residue $\bmod p$.

Thus for these "power of a primitive" one-relator groups, the homotopy classification for the Lens spaces $\mathscr{L}(p, q)$ for $\Xi$ is identical to that for the ordinary Lens spaces $L(p, q)$ for the cyclic group $Z_{p}$. We are able to solve the classification problem for the Lens spaces $\mathscr{L}(p, q)$ for the remaining one-relator group modulo this conjecture:

CONJECTURE. Let $\Xi$ be a one-relator group given by the presentation (1). If $R$ is not primitive in the free group $F$ generated by $x_{1}, \cdots, x_{n}$, then each automorphism $\alpha \in$ Aut $\Xi$ is induced by an automorphism of the free group $F$. (See S. Pride [7].)

THEOREM 2. Let $\Xi$ have presentation (1) with single relator $R^{p}$ a power of a nonprimitive element $R$ of the free group $F$. If the above conjecture holds for $\Xi$, then two Lens spaces $\mathscr{L}(p, q)$ and $\mathscr{L}(p, r)$ for $\Xi$ have the same homotopy type if and only if $q \equiv$ $\pm r \bmod p$.

The outline of this paper is as follows. In $\S 1$, we give the proofs of Theorems 1 and 2. In $\S 2$, we discuss the automorphisms of one-relator groups and give several examples for which the above conjecture holds. Finally, in $\S 3$, we discuss the non-cancellation aspect of these Lens spaces.

§o. Notation. For simplicity, we employ the same notation for elements of $F$ and $\Xi$. We let $Z \Xi$ denote the integral group ring of $\Xi$. All $Z \Xi$-modules are left $Z \Xi$-modules. Any element $w \in$ $Z \Xi$ defines a left $Z \Xi$-module homomorphism $w: Z \Xi \rightarrow Z \Xi$ given by the right multiplication. For $w \in \Xi$ and a positive integer $s$, we let $\langle w, s\rangle=1+w+\cdots+w^{s-1}$ and $\langle w,-s\rangle=-w^{-s}\langle w, s\rangle$ in $Z \Xi$. We have the following $\langle>$-identities

$$
\begin{aligned}
& (w-1)\langle w, s\rangle=w^{s}-1, \quad\langle w, s\rangle+w^{s}\langle w, t\rangle=\langle w, s+t\rangle, \\
& \langle w, s\rangle\left\langle w^{s}, t\right\rangle=\langle w, s t\rangle .
\end{aligned}
$$


If $w \in \Xi$ has order $p$, then $\langle w, s\rangle\langle w, p\rangle=s\langle w, p\rangle$.

All the spaces in this paper are connected $C W$-complexes with some zero cell chosen as basepoint which is preserved by all maps and homotopies.

1. Proofs. Throughout this paper $\Xi$ will be a one-relator group given by presentation (1). We denote by $S$ the element $\langle R, p\rangle=$ $1+R+\cdots+R^{p-1}$ of the integral group ring $Z \Xi$.

The following is a $\Xi$-resolution of the trivial $E$-module $Z$ (see R. Lyndon [4]):

$$
\begin{gathered}
\cdots \stackrel{S}{\longrightarrow} Z \Xi \stackrel{R-1}{\longrightarrow} Z \Xi \stackrel{S}{\longrightarrow} Z \Xi \stackrel{R-1}{\longrightarrow} Z \Xi \\
\stackrel{\partial_{2}}{\longrightarrow}(Z \Xi)^{n} \stackrel{\partial_{1}}{\longrightarrow} Z \Xi \stackrel{\varepsilon}{\longrightarrow} Z \longrightarrow 0
\end{gathered}
$$

where $\varepsilon: Z \Xi \rightarrow Z$ is the augmentation homomorphism,

$$
\partial_{1}=\left(x_{1}-1, \cdots, x_{n}-1\right) \text {, and } \partial_{2}=S\left(\partial R / \partial x_{1}, \cdots, \partial R / \partial x_{n}\right) \text {. }
$$

To the Lens space $\mathscr{L}(p, q)$ for $\Xi$, we can associate its algebraic 3-type $T(\mathscr{L}(p, q))=(\Xi, Z \Xi S, k)$ where $\pi_{1} \mathscr{L}(p, q)=\Xi, \pi_{3}(\mathscr{L}(p, q))=Z \Xi S$ and $k \in H^{4}(\Xi, Z \Xi S)$ is the obstruction invariant of MacLane-Whitehead [5]. An isomorphism

$$
(\Phi, \Psi): T(\mathscr{L}(p, q)) \longrightarrow T(\mathscr{L}(p, r))=\left(\Xi, Z \Xi S, k^{\prime}\right)
$$

between the algebraic 3-types consists of a group isomorphism $\Phi$ : $\Xi \rightarrow \Xi$ and a $Z \Xi$-module isomorphism $\Psi: Z \Xi S \rightarrow{ }_{\phi} Z \Xi S$ for which $\Phi^{*}\left(k^{\prime}\right)=\Psi_{*}(k)$ in the diagram:

$$
H^{4}(\Xi, Z \Xi S) \stackrel{{ }^{*} \Psi}{\longrightarrow} H^{4}\left(\Xi,{ }_{\phi} Z \Xi S\right) \stackrel{\Phi^{*}}{\longleftarrow} H^{4}(\Xi, Z \Xi S) .
$$

Note that we view ${ }_{\phi} Z E S$ as a $Z \Xi$-module this way: $w \cdot \alpha=\Phi(w) \alpha$ where $w \in Z \Xi$ and $\alpha \in Z \Xi S$. It is known that two Lens spaces $\mathscr{L}(p, q)$ and $\mathscr{L}(p, r)$ are homotopically equivalent if and only if $T(\mathscr{L}(p, q))$ is isomorphic to $T(\mathscr{L}(p, r)$ ). (See Theorems 4 and 5 of MacLaneWhitehead [5] and Theorem 15 of Whitehead [12]).

The cellular chain complex $C_{*}(\tilde{\mathscr{L}}(p, q))$ provides us with a partial free resolution $\varepsilon: C_{*}(\tilde{\mathscr{L}}(p, q)) \rightarrow Z$ which we can extend to get a free resolution $\varepsilon: C_{*}(\Xi) \rightarrow Z$ of the trivial module $Z$ over $Z \Xi$ (see [3]). Likewise we also obtain the free resolution $\varepsilon: \bar{C}_{*}(\Xi) \rightarrow Z$ of the trivial module $Z$ over $Z \Xi$ by extending the partial free resolution $\varepsilon: C_{*}(\tilde{\mathscr{L}}(p, r)) \rightarrow Z$. Let $u$ be any chain map

$$
u: C_{*}(\Xi) \longrightarrow \bar{C}_{*}(\Xi)
$$

extending the identity map on $Z$. Thus we have the commutative diagram: 


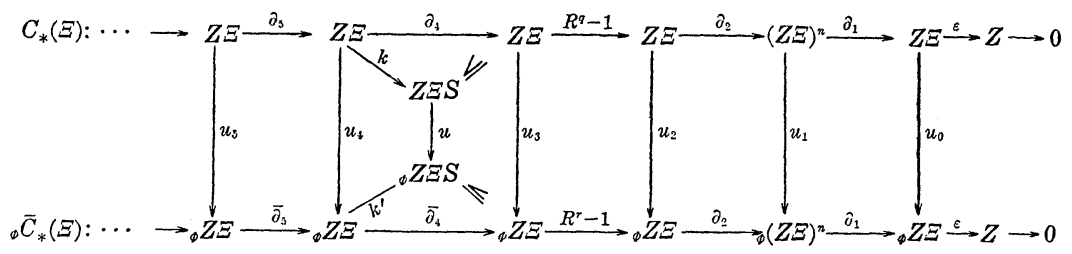

where $u$ also denotes the restriction of the chain map $u_{3}$ to $Z \Xi S$. The commutativity relation $u_{3} \partial_{4}=\bar{\partial}_{4} u_{4}$ implies that $u_{*}(k)=\Phi^{*}\left(k^{\prime}\right)$. (See [1].) Therefore $\mathscr{L}(p, q)$ and $\mathscr{L}(p, r)$ are homotopically equivalent if and only if for some chain map $u, u=u_{3} \mid Z \Xi S: Z \Xi S \rightarrow, Z \Xi S$ is a $Z \Xi$-module isomorphism; for then $(\Phi, u)$ constitutes an isomorphism of their algebraic 3-types.

We recall now the following two results of $R$. H. Fox [2] which are crucial to the following lemma.

I. Fundamental formula of free calculus.

Let $F$ denote the free group generated by $x_{1}, \cdots, x_{n}$ and let $v \in Z F$. Then

$$
\sum_{i=1}^{n} \frac{\partial v}{\partial x_{j}}\left(x_{j}-1\right)=v-\varepsilon(v)
$$

where $\varepsilon: Z F \rightarrow Z$ is the augmentation map.

II. Chain rule of differentiation.

Let $\lambda$ be a homomorphism from a free group $Y$ into a free group $X$. Then, for any $v \in Z Y$,

$$
\frac{\partial}{\partial x_{j}} \lambda(v)=\sum_{k} \lambda\left(\frac{\partial v}{\partial y_{k}}\right) \frac{\partial}{\partial x_{j}} \lambda\left(y_{k}\right) \text {. }
$$

LEMmA 1. Let $\Phi: \Xi \rightarrow \Xi$ be a group homomorphism such that $\Phi(R)=R^{s},(s, p)=1$. Then the following commutes:

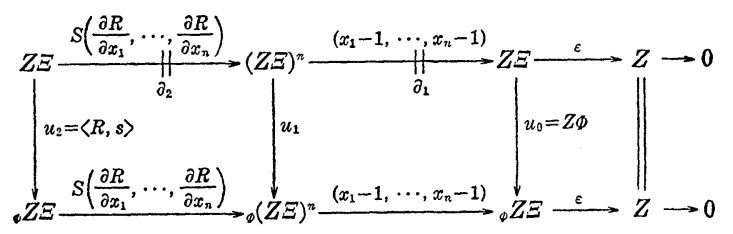

where

$$
u_{1}=\left(\begin{array}{ccc}
\frac{\partial \Phi\left(x_{1}\right)}{\partial x_{1}} & \cdots & \frac{\partial \Phi\left(x_{1}\right)}{\partial x_{n}} \\
\vdots & & \vdots \\
\frac{\partial \Phi\left(x_{n}\right)}{\partial x_{1}} & \cdots & \frac{\partial \Phi\left(x_{n}\right)}{\partial x_{n}}
\end{array}\right)_{n \times n .}
$$


Proof. To see that $u_{0} \partial_{1}=\partial_{1} u_{1}$, we consider the $i$ th column of the matrix $\partial_{1} u_{1}$ :

$$
\frac{\partial}{\partial x_{1}} \Phi\left(x_{i}\right)\left(x_{1}-1\right)+\cdots+\frac{\partial}{\partial x_{n}} \Phi\left(x_{i}\right)\left(x_{n}-1\right)
$$

$=\Phi\left(x_{i}\right)-1$ [by the fundamental formula of free calculus], which is the $i$ th column of the matrix $u_{0} \partial_{1}$. That $u_{1} \partial_{2}=\partial_{2} u_{2}$ follows from the relations

$$
\begin{aligned}
u_{1} \partial_{2}= & \Phi(S)\left[\Phi\left(\frac{\partial R}{\partial x_{1}}\right)\left(\frac{\partial}{\partial x_{1}} \Phi\left(x_{1}\right), \cdots, \frac{\partial}{\partial x_{n}} \Phi\left(x_{1}\right)\right)+\cdots\right. \\
& \left.\cdots+\Phi\left(\frac{\partial R}{\partial x_{n}}\right)\left(\frac{\partial}{\partial x_{1}} \Phi\left(x_{n}\right), \cdots, \frac{\partial}{\partial x_{n}} \Phi\left(x_{n}\right)\right)\right] \\
= & S\left(\Phi\left(\frac{\partial R}{\partial x_{1}}\right) \frac{\partial}{\partial x_{1}} \Phi\left(x_{1}\right)+\cdots+\Phi\left(\frac{\partial R}{\partial x_{n}}\right) \frac{\partial}{\partial x_{1}} \Phi\left(x_{n}\right), \cdots\right. \\
& \left.\cdots, \Phi\left(\frac{\partial R}{\partial x_{1}}\right) \frac{\partial}{\partial x_{n}} \Phi\left(x_{1}\right)+\cdots+\Phi\left(\frac{\partial R}{\partial x_{n}}\right) \frac{\partial}{\partial x_{n}} \Phi\left(x_{n}\right)\right) \\
= & S\left(\frac{\partial}{\partial x_{1}} \Phi(R), \cdots, \frac{\partial}{\partial x_{n}} \Phi(R)\right) \quad[\text { by chain rule] } \\
= & S\left(\frac{1}{\partial x_{1}} R^{s}, \cdots, \frac{\partial}{\partial x_{n}} R^{s}\right) \\
= & \langle R, s\rangle S\left(\frac{\partial R}{\partial x_{1}}, \cdots, \frac{\partial R}{\partial x_{n}}\right) \\
= & \partial_{2} u_{2} . \quad \text { This completes the proof. }
\end{aligned}
$$

The above lemma can be used to simplify the proof of Theorem 1 of [3].

Next we show that all inner automorphisms of $\Xi$ are contained in the image of the evaluation homomorphism $\#: \mathscr{E}(\mathscr{L}(p, q)) \rightarrow$ Aut $\Xi$ where $\mathscr{E}(\mathscr{L}(p, q))$ is the self-equivalence group of the Lens space $\mathscr{L}(p, q)$ for $\Xi$.

LEMMA 2. Let $\Phi$ be an inner automorphism of the one-relator group $\Xi$ given by presentation (1). Then $\Phi \in \sharp(\mathscr{E}(\mathscr{L}(p, q)))$.

Proof. We may assume that $\Phi(g)=x_{1} g x_{1}^{-1}$ for all $g$ in $\Xi$. Then we have the diagram:

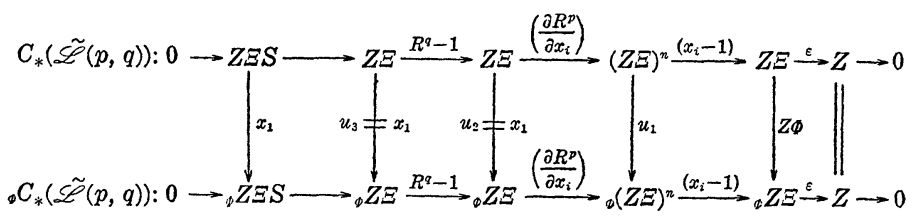


where $u_{1}$ is as in Lemma 1. Again, using the chain rule, one can show that $u_{1}$ and $u_{2}$ make the diagram commute. Because the multiplication $x_{1}: Z \Xi S \rightarrow{ }_{\phi} Z \Xi S$ by the group element $x_{1}$ is clearly an isomorphism, there is a self-homotopy equivalence $f: \mathscr{L}(p, q) \rightarrow \mathscr{L}(p, q)$ inducing the inner automorphism $f_{\#}=\Phi$ on the fundamental group (see the preliminary remarks at the begining of this section).

Proposition 1. Let $\Phi: \Xi \rightarrow \Xi$ be an automorphism. Then there is a positive integer $s$ such that $\Phi(R)=g R^{s} g^{-1}$ where $g \in \Xi$ and $(p, s)=1$. Moreover there exists a homotopy equivalence

$$
\mathscr{L}(p, q) \longrightarrow \mathscr{L}\left(p, q s^{2}\right)
$$

inducing $\Phi$ on the fundamental groups.

Proof. By Theorem 4.13 on page 269 of Magnus, Karrass, and Solitar [6], there is a positive integer $s$ such that $\Phi(R)=g R^{s} g^{-1}$; moreover, $(p, s)=1$ since $\Phi(R)$ has order $p$. Since Lemma 2 handles the problem of conjugation, we may assume that $\Phi(R)=R^{s}$ and so we have $\Phi(S)=S$. Thus in view of Lemma 1 , we have the commutative diagram

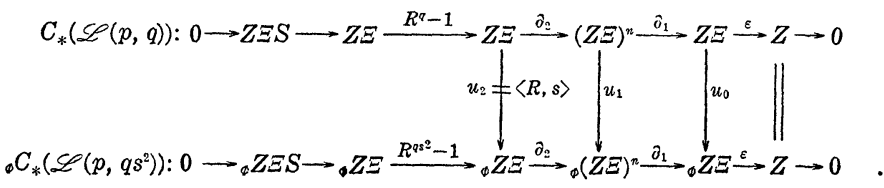

Because $\left(q s^{2}, p\right)=1$, there exist integers $a$ and $b$ such that $a q s^{2}+$ $b p=1$. We let $u_{3}=\langle R, q s\rangle\langle R, s\rangle\left\langle R^{q s^{2}}, a\right\rangle$ which makes the square commute since

$$
\begin{aligned}
\Phi\left(R^{q}-1\right)\langle R, s\rangle & =\left(R^{q s}-1\right)\langle R, s\rangle \\
& =\langle R, q s\rangle\langle R, s\rangle(R-1)
\end{aligned}
$$

and

$$
\begin{aligned}
\langle R, q s\rangle & \langle R, s\rangle\left\langle R^{q s^{2}}, a\right\rangle\left(R^{q s^{2}}-1\right) \\
& =\langle R, q s\rangle\langle R, s\rangle\left\langle R^{q s^{2}}, a\right\rangle\left\langle R, q s^{2}\right\rangle(R-1) \\
& =\langle R, q s\rangle\langle R, s\rangle\left\langle R, a q s^{2}\right\rangle(R-1) \\
& =\langle R, q s\rangle\langle R, s\rangle(R-1) .
\end{aligned}
$$

Let $b: Z \Xi \rightarrow{ }_{\phi} Z \Xi S$ be the $Z \Xi$-module homomorphism given by $1 \rightarrow b S$. Then $u_{3}+b$ restricts to $Z \Xi S$ to give the $Z \Xi$-module homomorphism $u: Z \Xi S \rightarrow{ }_{\Phi} Z \Xi S$ which maps $1 \cdot S \rightarrow\left(a q s^{2}+b p\right) S=1 \cdot S$; so $u$ is an isomorphism. Thus $u_{0}=Z \Phi, u_{1}, u_{2}$, and $u_{3}+b$ constitute a chain map $C_{*}(\tilde{\mathscr{L}}(p, q)) \rightarrow C_{*}\left(\tilde{\mathscr{L}}\left(p, q s^{2}\right)\right)$ which restricts to give an iso- 
morphism on the third homotopy module $Z \Xi S$. Hence there is a homotopy equivalence $f: \mathscr{L}(p, q) \rightarrow \mathscr{L}\left(p, q s^{2}\right)$ inducing $\Phi$ on the fundamental group.

Proposition 2. Two Lens spaces $\mathscr{L}(p, q)$ and $\mathscr{L}(p, r)$ for $\Xi$ are homotopically equivalent via the identity homomorphism on the fundamental groups if and only if $q \equiv \pm r \bmod p$.

Proof. First we assume that $\mathscr{L}(p, q)$ and $\mathscr{L}(p, r)$ are homotopically equivalent via the identity homomorphism on the fundamental groups. Because $(r, p)=1$, we may choose an integer $r^{\prime}$ such that $r r^{\prime} \equiv 1 \bmod p$. Then we have the diagram

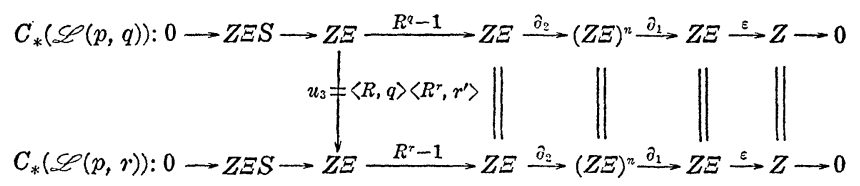

which commutes. Because $\mathscr{L}(p, q)$ and $\mathscr{L}(p, r)$ have the same homotopy type, there is a $Z \Xi$-homomorphism $\gamma: Z \Xi \rightarrow Z \Xi S$ such that $u_{3}+\gamma: Z \Xi \rightarrow Z \Xi$ restricts to $Z \Xi S$ to give an isomorphism $u: Z \Xi S \rightarrow$ $Z \Xi S . \quad \gamma$ is given by the formula (3.5) in Eilenberg-Maclane [1]. Now we have commutative diagram

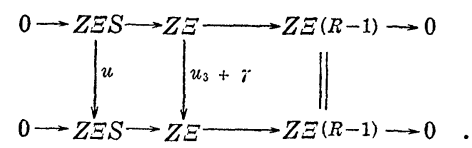

Therefore by Five lemma, $u_{3}+\gamma$ is an isomorphism. But then $\varepsilon\left(u_{3}+\gamma\right)=q r^{\prime}+p \varepsilon(v)= \pm 1$ for some $v \in Z \Xi$. Therefore $q r^{\prime} \equiv$ $\pm 1 \bmod p$, i.e., $q \equiv \pm r \bmod p$.

Conversely, let us assume that $q \equiv \pm r \bmod p$. We may choose an integer $k$ such that $k p=q \mp r$. Also, because $(r, p)=1$, there exist integers $r^{\prime}$ and $p^{\prime}$ such that $r r^{\prime}+p p^{\prime}=1$. As above, we may take the chain map $u_{3}=\langle R, q\rangle\left\langle R^{r}, r^{\prime}\right\rangle$, and let $-k r^{\prime} \pm p^{\prime}: Z \Xi \rightarrow Z \Xi S$ be the $Z \Xi$-module homomorphism given by $1 \rightarrow\left(-k r^{\prime} \pm p^{\prime}\right) S$. Then $u_{3}+\left(-k r^{\prime} \pm p^{\prime}\right)$ restricts to $Z \Xi S$ to give $Z \Xi$-module homomorphism which takes $1 \cdot S \rightarrow\left(q r^{\prime}-k r^{\prime} p \pm p^{\prime} p\right) S= \pm 1 S$, so that this restriction is an isomorphism. Therefore by the preliminary remarks at the start of this section, $\mathscr{L}(p, q)$ and $\mathscr{L}(p, r)$ have the same homotoy type.

In our attempts towards proving the general classification we are faced with the following problem: 
Given any integer $s$, relatively prime to $p$, does there exist an automorphism $\Phi \in$ Aut $\Xi$ with $\Phi(R)=R^{s}$ ?

In $\S 2$, we show that if the single-relator $R^{p}$ is power of a primitive, the answer to the above question is in the affimative. This allows us to "shuffle" the $k$-invariants of the Lens space $\mathscr{L}(p, q)$ for $\Xi$, and the classification for these Lens spaces is identical to that for the ordinary Lens spaces $L(p, q)$ (Theorem 1 ). On the other hand, if the single-relator $R^{p}$ is not power of a primitive, Pride conjectures that every $\Phi \in$ Aut $\Xi$ is free in which case we show that $\Phi(R)=g R^{ \pm 1} g^{-1}$ for some $g$ in $\Xi$. Thus we do not have the freedom of "shuffiing" the $k$-invariants; and so the solution to the general problem of homotopy classification for these Lens spaces is finer than that for the ordinary Lens spaces. Indeed it is identical to one where we insist on the identity homomorphism on the fundamental group (see Proposition 2 and Theorem 2).

Proposition 3. If there is an automorphism $\Phi \in$ Aut $\Xi$ with $\Phi(R)=g R^{s} g^{-1}$ where $(s, p)=1$ and $g \in \Xi$, then there is a homotopy equivalence between the Lens spaces $\mathscr{L}(p, q)$ and $\mathscr{L}(p, r)$ for $\Xi$ inducing $\Phi$ on the fundamental group if and only if $q s^{2} \equiv \pm r \cdot \bmod p$.

Proof. By Proposition 1, there is a homotopy equivalence between $\mathscr{L}(p, q)$ and $\mathscr{L}(p, r)$ inducing $\Phi$ on the fundamental group if and only if there is a homotopy equivalence between $\mathscr{L}\left(p, q s^{2}\right)$ and $\mathscr{L}(p, r)$ inducing the identity on the fundumental group and, by Proposition 2, this is the case if and only if $q s^{2} \equiv \pm r \bmod p$. We are done.

THEOREM 1. Let $\Xi$ have presentation (1) with the single-relator $R^{p}$ a power of a primitive element $R$ of $F$. Then two Lens spaces $\mathscr{L}(p, q)$ and $\mathscr{L}(p, r)$ for $\Xi$ have the same homotopy type if and only if $q r$ or $-q r$ is a quadratic residue $\bmod p$.

Proof. We first note that since $R$ is primitive, given any $s$, relatively prime to $p$, there is an automorphism $\Phi \in$ Aut $\Xi$ such that $\Phi(R)=R^{s}$ (see $\S 2$ ). Thus, by Proposition $3, \mathscr{L}(p, q)$ and $\mathscr{L}(p, r)$ have the same homotopy type if and only if for some $s,(s, p)=1$, we have that $q s^{2} \equiv \pm r \bmod p$, i.e., $(q s)^{2} \equiv \pm q r \bmod p$, and this is the case if and only if $q r$ or $-q r$ is a quadratic residue $\bmod p$.

THEOREM 2. Let $\Xi$ have presentation (1) with single relator $R^{p}$ a power of a nonprimitive element $R$ of the free group $F$. When conjecture stated in the introduction holds for $\Xi$, then two Lens spaces $\mathscr{L}(p, q)$ and $\mathscr{L}(p, r)$ have the same homotopy type if and 
only if $q \equiv \pm r \bmod p$.

Proof. Because $R$ is a nonprimitive element of the free group $F$, the conjecture gives that for any automorphism $\Phi \in$ Aut $\Phi$, $\Phi(R)=g R^{ \pm 1} g^{-1}, g \in \Xi$ (see $\left.\S 2\right)$. The theorem now is immediate from Proposition 3.

\section{Automorphisms of one-relator groups.}

Definition. An automorphism $\alpha: \Xi \rightarrow \Xi$ is free on a presentation $\mathscr{R}$ for $\Xi$ if it can be induced by an isomorphism on the free group on the generators of $\mathscr{R}$.

Lemma 11. Let $\alpha: \Xi \rightarrow \Xi$ be a free automorphism on the presentation $\mathscr{R}$ for $\Xi$. Then $\alpha(R)=g R^{ \pm 1} g^{-1}$ for some $g$ in $\Xi$.

Proof. By Theorem N5 of [6], page 172 (see also A. J. Sieradski [10, page 91]), we have that $\alpha\left(R^{p}\right)$ is freely equal (as a word in $x_{i}$ ) to $g R^{ \pm p} g^{-1}$ for some $g$ in $\Xi$. This implies that $\alpha(R)^{p}$ is freely equal $\left(g R^{ \pm 1} g^{-1}\right)^{p}$. But then by Exercise 2, page 41 of [6], $\alpha(R)=$ $g R^{ \pm 1} g^{-1}$.

Thus if $\alpha: \Xi \rightarrow \Xi$ is an automorphism for $\Xi$ with $\alpha(R)=R^{s}$ where $s \neq \pm 1$, then $\alpha$ is not free.

ExAMPLES. (1) G. Rosenberger in [9] has shown that every automorphism $\alpha$ of the group $H_{g, n}$ given by the presentation

$$
\left(a_{1}, \cdots, a_{g}, t_{1}, u_{1}, \cdots, t_{n} u_{n}:\left(a_{1}^{n_{1}} \cdots a_{g}^{n_{g}} \prod_{i=1}^{n}\left[t_{i}, u_{i}\right]\right)^{p}\right)
$$

$\left(p \geqq 2, n_{j} \geqq 2\right.$, for $\left.j=1, \cdots, g\right)$ is free.

(2) Let $C_{n, p}$ be the group presented by

$$
\mathscr{P}=\left(a_{1}, \cdots, a_{n-1}, c: c^{p}\right) .
$$

For any integer $s$ such that $(s, p)=1$, there is an automorphism $\alpha$ of $c_{n, p}$ with $\alpha_{s}(c)=c^{s}$. This is simply given by taking $a_{i} \rightarrow a_{i}$, $i=1, \cdots, n-1$, and $c \rightarrow c^{s}$. In fact the following example generalizes this situation.

(3) Let $\Xi$ be a group given by presentation (1). If $R$ is primitive in the free group $F$ generated by $x_{1}, \cdots, x_{n}$, then for all $s,(s, p)=1$, there is an automorphism $\alpha$ of $\Xi$ such that $\alpha(R)=R^{s}$. This follows because $R$ primitive implies that $E$ is isomorphic to $C_{n, p}$, and therefore Aut $\Xi$ is isomorphic to Aut $C_{n, p}$. Now it is easy 
to get a required $\alpha$.

(4) Let $\pi$ be the group presented by

$$
\left(x_{1}, x_{2}: Q^{q}\right)
$$

where $Q$ is not a proper power and $q>1$. Suppose also that $Q$ is not primitive in the free group $F$ generated by $x_{1}, x_{2}$. Then for any $\alpha$ in Aut $\pi, \alpha(Q)=g Q^{ \pm 1} g^{-1}$ for some $g$ in $\pi$. This follows from a result of S. Pride [8] which states that $\pi$ has only one Nielsen equivalence class.

3. Non-cancellation penomenon. In this section we show that although two Lens spaces $\mathscr{L}(p, q)$ and $\mathscr{L}(p, r)$ for $\Xi$ may have distinct homotopy type, $\mathscr{L}(p, q) \vee S^{3}$ and $\mathscr{L}(p, r) \vee S^{3}$ are always homotopically equivalent.

THEOREM. For every pair of Lens spaces $\mathscr{L}(p, q)$ and $\mathscr{L}(p, r)$ for $\Xi$, there is a homotopy equivalence

$$
\mathscr{L}(p, q) \vee S^{3} \longrightarrow \mathscr{L}(p, r) \vee S^{3}
$$

inducing the identity on the fundamental group.

Proof. We will show that the theorem holds for the pair $\mathscr{L}(p, q)$ and $\mathscr{L}(p, 1)$. Because $q$ and $p$ are relatively prime, there exists positive integers $q^{\prime}$ and $r^{\prime}$ such that $q q^{\prime}+p p^{\prime}=1$. We have the commutative diagram

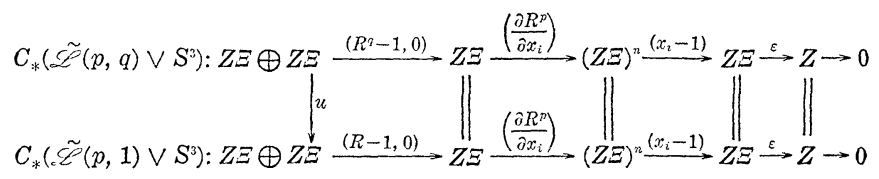

where $u=\left(\begin{array}{cc}\langle R, q\rangle & p^{\prime} \\ -\langle R, p\rangle & \left\langle R^{q}, q^{\prime}\right\rangle\end{array}\right)$. Clearly $u$ is an isomorphism with inverse $u^{-1}=\left(\begin{array}{cc}\left\langle R^{q}, q^{\prime}\right\rangle & -p^{\prime} \\ \langle R, p\rangle & \langle R, q\rangle\end{array}\right)$. Hence we can construct a homotopy equivalence between $\mathscr{L}(p, q)$ and $\mathscr{L}(p, 1)$ inducing the identity on the fundamental group.

\section{REFERENCES}

1. S. Eilenberg and S. MacLane, Homology of spaces with operators. II, Trans. Amer. Math. Soc., 65 (1949), 49-99.

2. R. H. Fox, Free differential calculus. I, Ann. Math., 57 (1953), 547-560.

3. S. Jajodia, On 2-dimensional $C W$-complexes with a single 2-cell, Pacific J. Math., 80 (1971), 191-203. 
4. R. Lyndon, Cohomology theory of groups with a single defining relation, Ann. Math., 52 (1950), 650-665.

5. S. MacLane and J. H. C. Whitehead, On the 3-type of a complex, Proc. Nat. Acad. Sci. U.S.A., 36 (1950), 41-48.

6. W. Magnus, A. Karras, and D. Solitar, Combinatorial Group Theory, Interscience, New York, 1966.

7. S. J. Pride, On the generation of one-relator groups, Trans. Amer. Math. Soc., 210 (1975), 331-364.

8. - The isomorphism problem for two-generator one-relator groups with torsion is solvable, to appear in Trans Amer. Math. Soc.

9. G. Rosenberger, Zum isomorphis problem für gruppen mit einer definierenden relation, Illinois. J. Math., 20 (1976), 614-621.

10. A. J. Sieradski, Combinatorial isomorphisms and combinatorial homotopy equivalences, J. Pure and Appl. Alg., 7 (1976), 59-95.

11. J. H. C. Whitehead, On incidence matrices, nuclei, and homotopy types, Ann. Math., 42 (1941), 1197-1239.

12. - Combinatorial homotopy. II, Bull. Amer. Math. Soc., 55 (1949), 453-496.

Received July 19, 1978 and in revised form July 6, 1979. Part of this work appeared in the thesis submitted by the author in partial fulfillment of the requirements for the $\mathrm{Ph}$. D. at the University of Oregon. The author wishes to thank his advisor Professor Allan J. Sieradski and Professor Micheal N. Dyer for their guidance and valuable advice.

University of Wisconsin-Stevens Point

Stevens PoInt, WI 54481 



\section{PACIFIC JOURNAL OF MATHEMATICS}

\section{EDITORS}

DONALD BABBITT (Managing Editor)

University of California

Los Angeles, CA 90024

HUGo RossI

University of Utah

Salt Lake City, UT 84112

C. C. MOORE and ANDREW OGG

University of California

Berkeley, CA 94720
J. DugundJI

Department of Mathematics

University of Southern California

Los Angeles, CA 90007

R. FinN and J. Milgram

Stanford University

Stanford, CA 94305

ASSOCIATE EDITORS
E. F. BECKENBACH
B. H. NeUmanN
F. WOLF
K. YoSHIDA

\section{SUPPORTING INSTITUTIONS}

UNIVERSITY OF BRITISH COLUMBIA

CALIFORNIA INSTITUTE OF TECHNOLOGY

UNIVERSITY OF CALIFORNIA

MONTANA STATE UNIVERSITY

UNIVERSITY OF NEVADA, RENO

NEW MEXICO STATE UNIVERSITY

OREGON STATE UNIVERSITY

UNIVERSITY OF OREGON
UNIVERSITY OF SOUTHERN CALIFORNIA

STANFORD UNIVERSITY

UNIVERSITY OF HAWAII

UNIVERSITY OF TOKYO

UNIVERSITY OF UTAH

WASHINGTON STATE UNIVERSITY

UNIVERSITY OF WASHINGTON

The Supporting Institutions listed above contribute to the cost of publication of this Journal, but they are not owners or publishers and have no responsibility for its content or policies.

Mathematical papers intended for publication in the Pacific Journal of Mathematics should be in typed form or offset-reproduced, (not dittoed), double spaced with large margins. Please do not use built up fractions in the text of the manuscript. However, you may use them in the displayed equations. Underline Greek letters in red, German in green, and script in blue. The first paragraph or two must be capable of being used separately as a synopsis of the entire paper. Please propose a heading for the odd numbered pages of less than 35 characters. Manuscripts, in triplicate, may be sent to any one of the editors. Please classify according to the scheme of Math. Reviews, Index to Vol. 39. Supply name and address of author to whom proofs should be sent. All other communications should be addressed to the managing editor, or Elaine Barth, University of California, Los Angeles, California, 90024.

50 reprints to each author are provided free for each article, only if page charges have been substantially paid. Additional copies may be obtained at cost in multiples of 50 .

The Pacific Journal of Mathematics is issued monthly as of January 1966. Regular subscription rate: $\$ 84.00$ a year (6 Vols., 12 issues). Special rato: $\$ 42.00$ a year to individual members of supporting institutions.

Subscriptions, orders for numbers issued in the last three calendar years, and changes of address shoud be sent to Pacific Journal of Mathematics, P.O. Box 969, Carmel Valley, CA 93924, U.S.A Old back numbers obtainable from Kraus Periodicals Co., Route 100, Millwood, NY 10546.

PUBLISHED BY PACIFIC JOURNAL OF MATHEMATICS, A NON-PROFIT CORPORATION

Printed at Kokusai Bunken Insatsusha (International Academic Printing Co., Ltd.). 8-8, 3-chome, Takadanobaba, Shinjuku-ku, Tokyo 160, Japan.

Copyright (C) 1980 by Pacific Jounal of Mathematics Manufactured and first issued in Japan 


\section{Pacific Journal of Mathematics \\ Vol. 89, No. $2 \quad$ June, 1980}

Frank Hayne Beatrous, Jr. and R. Michael Range, On holomorphic

approximation in weakly pseudoconvex domains................. 249

Lawrence Victor Berman, Quadratic forms and power series fields ...... 257

John Bligh Conway and Wacław Szymański, Singly generated antisymmetric operator algebras ....................... 269

Patrick C. Endicott and J. Wolfgang Smith, A homology spectral sequence for submersions . . . .................................

Sushil Jajodia, Homotopy classification of lens spaces for one-relator groups with torsion ................................ 301

Herbert Meyer Kamowitz, Compact endomorphisms of Banach

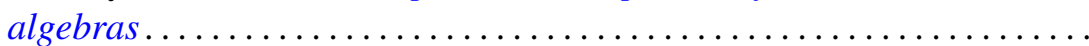

Keith Milo Kendig, Moiré phenomena in algebraic geometry: polynomial

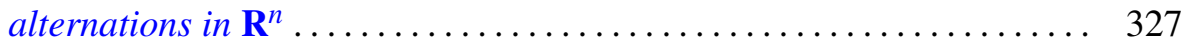

Cecelia Laurie, Invariant subspace lattices and compact operators....... 351

Ronald Leslie Lipsman, Restrictions of principal series to a real form . . . . . 367

Douglas C. McMahon and Louis Jack Nachman, An intrinsic

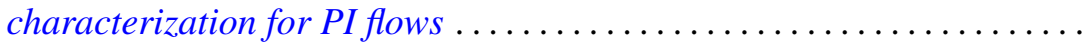

Norman R. Reilly, Modular sublattices of the lattice of varieties of inverse semigroups .................................... 405

Jeffrey Arthur Rosoff, Effective divisor classes and blowings-up of $\mathbf{P}^{2}$ 419

Zalman Rubinstein, Solution of the middle coefficient problem for certain

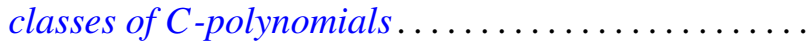

Alladi Sitaram, An analogue of the Wiener-Tauberian theorem for spherical transforms on semisimple Lie groups ................

Hal Leslie Smith, A note on disconjugacy for second order systems ...

J. Wolfgang Smith, Fiber homology and orientability of maps ...

Audrey Anne Terras, Integral formulas and integral tests for series of positive matrices. 